\title{
Numbers for reducible cubic scrolls
}

\author{
ISRAEL VAINSENCHER ${ }^{1}$ and FERNANDO XAVIER ${ }^{2}$ \\ ${ }^{1}$ ICEX - Departamento de Matemática - UFMG \\ Cx. Postal 702 Belo Horizonte 30123-970 Belo Horizonte, MG, Brasil \\ ${ }^{2}$ Departamento de Matemática - UFPb \\ Cidade Universitária - 58051-900 João Pessoa, PB, Brasil \\ Manuscript received on January 19, 2004; accepted for publication on July 6, 2004; \\ contributed by ISRAEL VAINSENCHER*
}

\begin{abstract}
We show how to compute the number of reducible cubic scrolls of codimension 2 in $\mathbb{P}^{n}$ incident to the appropriate number of linear spaces.
\end{abstract}

Key words: cubic scrolls, enumerative geometry.

\section{INTRODUCTION}

A cubic rational normal scroll of codimension 2 in $\mathbb{P}^{n}$ is the residual intersection of 2 quadric hypersurfaces containing a codimension 2 plane. It is also equal to the locus of rank 1 of a $2 \times 3$ matrix

$$
\left(\begin{array}{ccc}
L_{1}(x) & L_{2}(x) & L_{3}(x) \\
M_{1}(x) & M_{2}(x) & M_{3}(x)
\end{array}\right)
$$

(cf. (Harris 1992) where the $L_{i}, M_{j}$ denote sufficiently general linear forms in the homogeneous coordinates $x_{0}, \ldots, x_{n}$ of $\mathbb{P}^{n}$. For $n=3$, this is a twisted cubic. Enumeration of such curves has attracted some attention in the last two decades, and is now but an example in GromovWitten theory, cf. (Fulton and Pandharipande 1997), (Kock and Vainsencher 1999). No such general technique is known yet for higher dimension. For the case of cubic scrolls, a procedure to determine some of these numbers was explained in (Vainsencher and Xavier 2002).

The purpose of this note is to show an application of Bott's residual formula for the calculation of the number of reducible $(n-2)$-dimensional cubic scrolls in $\mathbb{P}^{n}$ satisfying suitable incidence

*Member Academia Brasileira de Ciências

Correspondence to: Israel Vainsencher

E-mail: israel@mat.ufmg.br 
conditions to linear subspaces. The whole point is the simple observation that, just as in the case of twisted cubics, a general reducible cubic scroll of dimension $(n-2)-$ is the union of an $(n-2)$ - plane with an $(n-2)$-dimensional quadric meeting along an $(n-3)$-plane.

\section{THE PARAMETER SPACE}

As customary in enumerative questions, we take the lead from the previous observation and consider the incidence variety,

$$
\mathbb{X}=\{(p, \ell, \kappa \subset h) \mid p=[n-3] \subset \ell=[n-2], p \subset \kappa=\text { quadric in the hyperplane } h\} .
$$

$\mathbb{X}$ is naturally obtained as a tower of fiber bundles. Start with the grassmannian,

$$
\mathbb{Y}:=\mathbb{G} r[n-3 n]
$$

of projective $(n-3)$-dimensional subspaces of $\mathbb{P}^{n}$. It carries a tautological exact sequence

$$
\mathcal{S} \longrightarrow \mathcal{F} \rightarrow \mathcal{Q}
$$

where $\operatorname{rank} \mathcal{S}=3$ and $\mathcal{F}$ denotes the trivial bundle with fiber the dual space $\left(\mathbb{C}^{n+1}\right)^{\star}$. The homogeneous coordinates $x_{0}, \ldots, x_{n}$ form a basis for the latter space. The fiber of $\mathcal{S}$ over $p=[n-3] \in \mathbb{Y}$ is the 3-dimensional space of linear forms vanishing on $p$. The choice of a space $\ell=[n-2]$ containing a fixed $p=[n-3]$ is tantamount to picking a 2-dimensional vector subspace of the fiber $\mathcal{S}_{p}$, i.e., a point in the dual space $\mathcal{S}_{p}^{\star}$. Thus, the set of pairs $p \subset \ell$ is the total space of the $\mathbb{P}^{2}-$ bundle, $\mathbb{P}\left(\mathcal{S}^{\star}\right) \rightarrow \mathbb{Y}$. Likewise, hyperplanes $h$ through $p$ form the total space of the $\mathbb{P}^{2}$-bundle, $\mathbb{P}(\mathcal{S}) \rightarrow \mathbb{Y}$. The choice of a quadric $\kappa \subset h$ containing $p$ yields the $\mathbb{P}^{2 n-2}-$ bundle,

$$
\mathbb{P}(\overline{\mathcal{R}}) \rightarrow \mathbb{P}(\mathcal{S})
$$

Here $\overline{\mathcal{R}}$ stands for the rank- $(2 n-1)$ vector bundle over $\mathbb{P}(\mathcal{S})$ obtained as follows. Let $\mathcal{R}$ be the kernel of $S_{2} \mathcal{F} \rightarrow S_{2} \mathcal{Q}$. Since $3 n=\left(\begin{array}{c}n+2 \\ 2\end{array}\right)-\left(\begin{array}{c}n-3+2 \\ 2\end{array}\right)$, this is the rank-3n bundle of quadrics vanishing at the varying $p \in \mathbb{Y}$. Let $\mathcal{H}=\mathcal{O}_{\mathcal{S}}(-1) \rightarrow \mathcal{S}$ be the line bundle over $\mathbb{P}(\mathcal{S})$ with fiber over $h$ given by the scalar multiples of the linear form $h$. Put $\overline{\mathcal{F}}=\mathcal{F} / \mathcal{H}$. Then $\overline{\mathcal{R}}$ fits into the diagram of natural vector bundle maps,

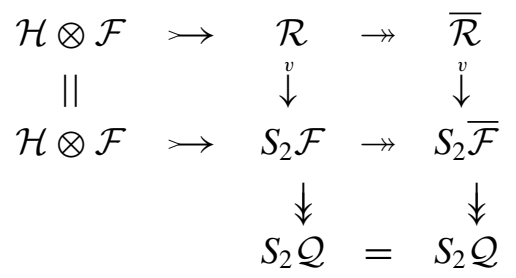

We may summarize the previous discussion as follows.

LEMma 2.1. Notation as above, the parameter space $\mathbb{X}$ is isomorphic to the fiber product,

$$
\mathbb{X}=\mathbb{P}\left(\mathcal{S}^{\star}\right) \times_{\mathbb{Y}} \mathbb{P}(\overline{\mathcal{R}})
$$

and $\operatorname{dim} \mathbb{X}=5 n-4$. 
REMARK 2.2. Let

$$
\widetilde{\mathbb{X}}=\left\{((p, \ell, \kappa), x) \in \mathbb{X} \times \mathbb{P}^{n} \mid x \in \kappa \cup \ell\right\} .
$$

It should be noted that $\widetilde{\mathbb{X}} \rightarrow \mathbb{X}$ is not a flat family. It can be rendered flat by a single blowup along a suitable smooth subvariety of $\mathbb{X}$ using techniques as in (Vainsencher and Xavier 2002), but this is not needed in the sequel.

\section{CYCLE CLASSES}

Now we define the divisors corresponding to the relevant incidence conditions.

Fix a line $\ell_{0}=\mathbb{P}^{1} \subset \mathbb{P}^{n}$ and a plane $\pi_{0}=\mathbb{P}^{2} \subset \mathbb{P}^{n}$. We define

$$
\begin{aligned}
& D_{\pi_{0}, p}=\left\{p \in \mathbb{Y} \mid p \cap \pi_{0} \neq \emptyset\right\} \\
& D_{\ell_{0}, \ell}=\left\{\ell \in \mathbb{P}\left(\mathcal{S}^{\star}\right) \mid \ell \cap \ell_{0} \neq \emptyset\right\} \\
& D_{\ell_{0}, \kappa}=\left\{\kappa \in \mathbb{P}(\overline{\mathcal{R}}) \mid \kappa \cap \ell_{0} \neq \emptyset\right\} .
\end{aligned}
$$

We also need a bit more of notation. We write

$$
\mathcal{L} \rightarrow \mathcal{S} \rightarrow \mathcal{O}_{\mathcal{S}^{\star}}(1)
$$

for the tautological sequence over $\mathbb{P}\left(\mathcal{S}^{\star}\right)$, where rank $\mathcal{L}=2$.

PROPOSITION 3.1. The cycle classes of the divisors defined above can be expressed as follows in terms of Chern classes of the natural bundles:

(1) $D_{\pi_{0}, p}=c_{1}(\mathcal{Q})$.

(2) $D_{\ell_{0}, \ell}=c_{1}\left(\mathcal{L}^{\star}\right)=c_{1}\left(\mathcal{S}^{\star}\right)+c_{1}\left(\mathcal{O}_{\mathcal{S}^{\star}}(1)\right)=c_{1}(\mathcal{Q})+c_{1}\left(\mathcal{O}_{\mathcal{S}^{\star}}(1)\right)$.

(3) $D_{\ell_{0}, \kappa}=2 c_{1}\left(\mathcal{O}_{\mathcal{S}}(1)\right)+c_{1}\left(\mathcal{O}_{\overline{\mathcal{R}}}(1)\right)$.

Proof. Let $\mathcal{E}=\left\langle x_{3}, \ldots, x_{n}\right\rangle \subset \mathcal{F}$ be the space of $n-2$ linear forms cutting $\pi_{0}=\mathbb{P}^{2} \subset \mathbb{P}^{n}$. Let $\overline{\mathcal{E}}=\mathcal{F} / \mathcal{E}$ denote the rank-3 quotient. Studying the diagram of vector bundles over $\mathbb{Y}$,

$$
\begin{array}{ccccc} 
& \mathcal{E} & & \\
& & \downarrow & & \\
\mathcal{S} & \longrightarrow & \mathcal{F} & \rightarrow & \mathcal{Q} \\
\sigma & \searrow & & & \\
& & & & \\
& & & &
\end{array}
$$

it can be seen that $p \in \mathbb{Y}$ meets $\pi_{0}$ if and only if the slant arrow $\sigma: \mathcal{S} \rightarrow \overline{\mathcal{E}}$ is not injective at $p$. Hence $D_{\pi_{0}, p}$ is the divisor of zeros of $\wedge^{\wedge} \sigma$. It follows that $D_{\pi_{0}, p}=c_{1}\left(\stackrel{3}{\wedge} \mathcal{S}^{\star}\right)=c_{1}(\mathcal{Q})$. The proof of 2 . is similar. The last formula is slightly trickier. It suffices to establish it over an open subset the complement of which contains no divisor. Thus, we may restrict to the locus of $h$ transversal to $\ell_{0}$. Let $\mathcal{E}=\left\langle x_{2}, \ldots, x_{n}\right\rangle \subset \mathcal{F}$ be now the space of equations for $\ell_{0}=\mathbb{P}^{1} \subset \mathbb{P}^{n}$. 
Let $\overline{\mathcal{E}}=\mathcal{F} / \mathcal{E}$ be the rank 2 quotient. The composition $\mathcal{H} \rightarrow \mathcal{F} \rightarrow \overline{\mathcal{E}} \simeq \mathbb{C}^{2}$ is injective over the transversal locus. The quotient map $\mathcal{F} \rightarrow \overline{\mathcal{H}}=\mathcal{F} /(\mathcal{E}+\mathcal{H})$ can be thought of as evaluation of linear forms at the point of intersecion of $\ell_{0}$ with a varying, transversal hyperplane. It yields an evaluation map for quadratic forms, $S_{2} \mathcal{F} \rightarrow S_{2} \overline{\mathcal{H}}$ and induces $S_{2}(\mathcal{F} / \mathcal{H}) \rightarrow S_{2} \overline{\mathcal{H}}$. Composing with $\mathcal{O}_{\overline{\mathcal{R}}}(-1) \longrightarrow \overline{\mathcal{R}} \longrightarrow S_{2}(\mathcal{F} / \mathcal{H})$, we find $\mathcal{O}_{\overline{\mathcal{R}}}(-1) \rightarrow S_{2} \overline{\mathcal{H}}$. The latter vanishes precisely over $D_{\ell_{0}, \kappa}$ (along the transversal locus). Since $c_{1}\left(S_{2} \overline{\mathcal{H}}\right)=2 c_{1}(\overline{\mathcal{H}})=-2 c_{1}(\mathcal{H})$, the formula follows.

\section{NUMBERS}

We shall abuse notation and keep writing

$$
D_{\pi_{0}, p}, D_{\ell_{0}, \ell}, D_{\ell_{0}, \kappa}
$$

for the pullback of these divisors to $\mathbb{X}$. The intersection of general translates is transversal by standard Bertini-Kleiman-Sard arguments (applied to (2.2) $\widetilde{\mathbb{X}} \rightarrow \mathbb{P}^{n}$ ). Taking $i_{1}+i_{2}+i_{3}=$ $5 n-4=\operatorname{dim} \mathbb{X}$ such translates, we may find the number of elements $(p, \ell, \kappa)$ of $\mathbb{X}$ such that the subspace $p=[n-3]$ meets $i_{1} \mathbb{P}^{2}$, s, the subspace $\ell=[n-2]$ meets $i_{2} \mathbb{P}^{1}$ 's and the $(n-2)$-quadric $\kappa$ meets $i_{3} \mathbb{P}^{1}$ 's. This is done by evaluating $N_{i_{1}, i_{2}}:=$

$$
\int_{\mathbb{X}}\left(D_{\pi_{0}, p}\right)^{i_{1}} \cdot\left(D_{\ell_{0}, \ell}\right)^{i_{2}} \cdot\left(D_{\ell_{0}, \kappa}\right)^{i_{3}}=\int_{\mathbb{X}} c_{1}(\mathcal{Q})^{i_{1}} \cdot c_{1}\left(\mathcal{L}^{\star}\right)^{i_{2}} \cdot\left(2 c_{1}\left(\mathcal{O}_{\mathcal{S}}(1)\right)+c_{1}\left(\mathcal{O}_{\overline{\mathcal{R}}}(1)\right)\right)^{i_{3}} .
$$

For the specific task of enumerating the reducible cubic scrolls, we take translates of the divisors $D_{\pi_{0}, p}$ and $D_{\ell_{0}, \ell \cup \kappa}:=D_{\ell_{0}, \ell}+D_{\ell_{0}, \kappa}$. We are now asked to compute

$$
N_{i}:=\int_{X}\left(D_{\pi_{0}, p}\right)^{i} \cdot\left(D_{\ell_{0}, \ell \cup \kappa}\right)^{j}
$$

for $i+j=\operatorname{dim} \mathbb{X}$. This is a purely mechanical matter with the help of either Schubert calculus (e.g., as implemented in (Katz and Strømme 1992) or Bott's residue formula (cf. (Bott 1967), (Meurer 1996)). Here are the ingredients for the latter. First find the fixed points of a $\mathbb{C}^{\star}-$ action on $\mathbb{X}$, induced by an action $x_{i} \mapsto t^{w_{i}} x_{i}$ on $\mathbb{P}^{n}$. Choosing the integral weights $w_{i}$ sufficiently general, it can be checked that all fixed points are isolated, starting at $\mathbb{Y}$, then climbing up the tower

$$
\mathbb{Y} \leftarrow \mathbb{P}(\mathcal{S}) \leftarrow \mathbb{P}(\overline{\mathcal{R}}) \leftarrow \mathbb{P}\left(\mathcal{S}^{\star}\right) \times_{\mathbb{Y}} \mathbb{P}(\overline{\mathcal{R}})=\mathbb{X}
$$

Pick $p_{1}=\left\langle x_{0}, x_{1}, x_{2}\right\rangle \in \mathbb{Y}=\mathbb{G} r[n-3 n]$, among the $\left(\begin{array}{c}n+1 \\ 3\end{array}\right)$ fixed points in $\mathbb{Y}$. There are just three fixed points on the fiber $\mathbb{P}^{2}=\mathbb{P}\left(\mathcal{S}_{p_{1}}\right)$. Choose one, say $p_{1,1}=\left\langle x_{0}\right\rangle$ (a hyperplane through $p_{1}$ ). The fiber of $\mathbb{P}(\overline{\mathcal{R}})$ over $p_{1,1}$ is

$$
\mathbb{P}^{2 n-2}=\mathbb{P}\left(\left\langle x_{1}^{2}, x_{1} x_{2}, \ldots, x_{1} x_{n}, x_{2}^{2}, \ldots, x_{2} x_{n}\right\rangle\right),
$$

with the obvious $2 n-1$ fixed points. Pick $p_{1,1,1}=\left\langle x_{1}^{2}\right\rangle$. By the same token, the fiber of $\mathbb{X} \rightarrow \mathbb{P}(\overline{\mathcal{R}})$ over $p_{1,1,1}$ is $\mathbb{P}^{2}=\mathbb{P}\left(\left\langle\bar{x}_{0}, \bar{x}_{1}, \bar{x}_{2}\right\rangle\right)$, with $\bar{x}_{i}=\left\langle x_{0}, x_{1}, x_{2}\right\rangle /\left\langle x_{j}, x_{k}\right\rangle$. We obtain thus the fixed point $p_{1,1,1,1}=\bar{x}_{0}$ (among a total of $\left(\begin{array}{c}n+1 \\ 3\end{array}\right) \cdot 3 \cdot(2 n-1) \cdot 3$ possible ones). The contribution of this fixed point 
to Bott's formula is given by evaluating the indicated Chern classes in the equivariant cohomology ring at the point. Explicitly, $c_{1}(\mathcal{Q})$ evaluates to $\sum_{3}^{n} w_{i}$, because the fiber $\mathcal{Q}_{p_{1}}=\left\langle x_{3}, \ldots, x_{n}\right\rangle$ decomposes into eigenspaces with weights $w_{i}, i=3 \ldots n$. The term $c_{1}\left(\mathcal{L}^{\star}\right)$ evaluates to $-w_{1}-w_{2}$. Next, the fiber of $\mathcal{O}_{\mathcal{S}}(-1)$ is the line spanned by the equation of the hyperplane $\left\langle x_{0}\right\rangle$; dualizing, we see that $c_{1}\left(\mathcal{O}_{\mathcal{S}}(1)\right)$ yields $-w_{0}$. The fiber of $\mathcal{O}_{\overline{\mathcal{R}}}(1)$ is dual to the line $\left\langle x_{1}^{2}\right\rangle$ hence contributes with $-2 w_{1}$. We also need the weights of the fiber of the tangent bundle of $\mathbb{X}$. Starting at $T_{p_{1}} \mathbb{Y}$, we write the eigenspace decomposition $\operatorname{Hom}\left(\left\langle x_{3}, \ldots, x_{n}\right\rangle, \mathcal{F} /\left\langle x_{3}, \ldots, x_{n}\right\rangle\right)=\sum x_{i} / x_{j}$, with $i \in\{0,1,2\}, j \in\{3, \ldots, n\}$. Add to this the (decomposition of the) tangent along the fiber of $\mathbb{P}(\mathcal{S}) \rightarrow \mathbb{Y}$, at $\left\langle x_{0}\right\rangle$, namely, $x_{1} / x_{0}+x_{2} / x_{0}$. Continuing, get the tangent along the fiber of $\mathbb{P}(\overline{\mathcal{R}}) \rightarrow \mathbb{P}(\mathcal{S})$, to wit, $\left(x_{1} x_{2}+\cdots+x_{1} x_{n}+x_{2}^{2}+\cdots+x_{2} x_{n}\right) / x_{1}^{2}$. At last, add the fiber of $T\left(\mathbb{P}\left(\mathcal{S}^{\star}\right) / \mathbb{Y}\right)$ at $\left\langle\bar{x}_{0}\right\rangle, x_{0} / x_{1}+x_{0} / x_{2}$. The top Chern class needed is the product of weights $\left(w_{i}-w_{j}\right), i \in\{0,1,2\}, j \in\{3, \ldots, n\}$, times $\left(w_{1}-w_{0}\right)\left(w_{2}-w_{0}\right)$, times $\left(w_{2}-w_{1}\right) \cdots\left(w_{n}-\right.$ $\left.w_{1}\right)\left(2 w_{2}-2 w_{1}\right) \cdots\left(w_{2}+w_{n}-2 w_{1}\right)$, times $\left(w_{0}-w_{1}\right)\left(w_{0}-w_{2}\right)$; call this product $w$. The total contribution of the present fixed point is the fraction

$$
\left(\left(\sum_{3}^{n} w_{i}\right)^{i_{1}}\left(-w_{1}-w_{2}\right)^{i_{2}}\left(-2 w_{0}-2 w_{1}\right)^{i_{3}}\right) / w .
$$

Quite miraculously, adding up the contributions over all fixed points, we get an integer.

The table below compiles some examples for $3 \leq n \leq 6$.

\begin{tabular}{|c|c|c||c|c|c|}
\hline $\mathrm{n}$ & $\mathrm{i}$ & $N_{i}$ & $i_{1}$ & $i_{2}$ & $N_{i_{1}, i_{2}}$ \\
\hline 3 & 0 & 121440 & 0 & 3 & 368 \\
\hline 3 & 1 & 37920 & 0 & 4 & 184 \\
\hline 3 & 2 & 6336 & 1 & 4 & 36 \\
\hline 3 & 3 & 504 & 2 & 2 & 92 \\
\hline 3 & 4 & 0 & 3 & 2 & 18 \\
\hline 4 & 0 & 285726000 & 4 & 2 & 5700 \\
\hline 4 & 3 & 7484880 & 5 & 2 & 1570 \\
\hline 5 & 0 & 681923574360 & & & \\
\hline 5 & 1 & 259044433830 & & & \\
\hline 6 & 0 & 1676746892620800 & & & \\
\hline
\end{tabular}

Thus we find in $\mathbb{P}^{4}$ the numbers $N_{3}=7484880$ of reducible cubic scrolls $\ell \cup \kappa \leftrightarrow(p, \ell, \kappa)$ such that the line $p=\mathbb{P}^{1} \subset \mathbb{P}^{4}$ meets 3 general given $\mathbb{P}^{2} \subset \mathbb{P}^{4}$ and $p \subseteq \ell \cap \kappa$ and the union $\ell \cup \kappa$ meets $\operatorname{dim} \mathbb{X}-3=5 \cdot 4-4-3=13$ general lines. On the same row of the table we read $N_{5,2}=1570$, the number of configurations $(p, \ell, \kappa) \in \mathbb{X}$ with the line $p$ meeting $i_{1}=5$ general $\mathbb{P}^{2}$ 's, the plane $\ell$ meeting $i_{2}=2$ general lines and the quadric surface $\kappa$ meeting $i_{3}=16-i_{1}-i_{2}=9$ other general lines. The entries have been computed employing Greuel et al. 2001. A script can be downloaded from Vainsencher 2004. 


\section{Closing Remarks}

As kindly pointed out by the referee, for $n>5$ all rational normal cubic scrolls of codimension 2 in $\mathbb{P}^{n}$ are cones (cf. XXX 1957). For $n=4,5$, the irreducible singular cubic scrolls not contained in a hyperplane are cones over a scroll in a hyperplane. The enumeration of such cones satisfying suitable incidence conditions can be pursued by means of a natural fibration. We hope to report on this elsewhere.

\section{ACKNOWLEDGMENTS}

We thank the referees for several suggestions and corrections. This work was partially supported by Conselho Nacional de Desenvolvimento Científico e Tecnológico (CNPq).

\section{RESUMO}

Mostramos como calcular o número de rolos cúbicos redutíveis de codimensão 2 em $\mathbb{P}^{n}$ incidentes a espaços lineares apropriados.

Palavras-chave: rolos cúbicos, geometria enumerativa.

\section{REFERENCES}

Bотт R. 1967. A residue Formula for Holomorphic Vector Fields. J Diff Geom 1: 311-330.

Fulton W and Pandharipande R. 1997. Notes on Stable Maps and Quantum Cohomology. Proc Symp Purê Math 62(II): 45-96 (alg-geom/9608011).

Greuel G-M, Pfister G and Schönemann H. 2001. Singular 2.0. A Computer Álgebra System for Polynomial Computations. Centre for Computer Álgebra, University of Kaiserslautern. http://www. singular.uni-kl.de.

HARRIS J. 1992. Algebraic geometry. A first course, Texts in Matematics, 133. Springer-Verlag, New York.

Katz S and Strømme SA. 1992. Schubert. A maple package for Intersection Theory. http://www. mi.uib.no/schubert/.

Kock J AND VAINSENCher I. 1999. Kontsevich's formula for rational plane curves, book, to appear, http://www.mat.ufmg.br/ israel/kontsevich.html, Portuguese original version: A fórmula de Kontsevich para curvas racionais planas, $22^{\circ}$ Colóquio Brasileiro de Matemática. [22nd Brazilian Mathematics Colloquium] (IMPA), Rio de Janeiro.

Meurer P. 1996. The number of rational quartics on Calabi-Yau hypersurfaces in weighted projective space P(2,14) Math Scandin 78: 63-83 (alg-geom/9409001).

VAinsencher I AND XAVIER F. 2002. A compactification of the space of twisted cubics. Math Scand 91: 221-243.

VAINSENCHER I. 2004. http://www.mat.ufmg.br/ israel/sing.html.

XXX (anOnymous) 1957. Correspondence. Am J Math 79: 951-952. 